\title{
Enhancing self-assembled colloidal quantum dot microsphere lasers
}

\author{
Pedro Urbano Alves, Dimitars Jevtics, Michael J. Strain, Martin D. Dawson, Nicolas Laurand \\ Institute of Photonics, Department of Physics, SUPA, University of Strathclyde \\ Glasgow, $U K$ \\ pedro.alves@strath.ac.uk
}

\begin{abstract}
We demonstrate the superior emission properties of a red-emitting self-assembled quantum dot microsphere laser made from a blend of green and red bandgap $\mathrm{CdS}_{\mathrm{x}} \mathrm{Se}_{1-\mathrm{x}} / \mathrm{ZnS}$ quantum dots. The addition of the higher bandgap material reduces self-absorption and improves the Q-factor.
\end{abstract}

Keywords - Quantum dots, lasers, nanocrystals, semiconductors, microresonators.

\section{INTRODUCTION}

An optimal design of spherical microresonators requires the tuning of several parameters to minimize the loss of stored energy [1]. In spherical supraparticles (SPs) - self-assembled microspheres of colloidal quantum dots (CQDs) - the parameters that determine their quality factor (Q-factor) not only depend on the shape, size, and average refractive index of the SP, but also on their building blocks - the CQDs. In the solid state, CQDs can suffer from self-absorption, exciton diffusion and nonradiative recombination [2]. In combination, these factors dampen the oscillations of whispering gallery modes and decrease their Q-factor and this has in turn a detrimental effect on the properties of SP lasers. In this work, the addition of higher bandgap CQDs to the fabrication of SPs is studied as a way of preventing such losses. A SP laser blending CQDs of two different bandgaps is shown to operate at higher intensity than, and with a similar threshold to, the equivalent structure without higher bandgap CQDs. Such SP lasers have great application potential for integrated circuits, imaging, sensing and novel photonic materials [3], [4].

\section{MATERIALS AND METHODOLOGY}

\section{A. Synthesis of the Supra Particles}

An oil-in-water emulsion technique [3], [4] was used to synthesize spherical SPs made of $\mathrm{CdS}_{\mathrm{x}} \mathrm{Se}_{1-\mathrm{x}} / \mathrm{ZnS} \mathrm{CQDs}$ with oleic acid ligands. The CQDs were approximately $6.0 \pm 0.5 \mathrm{~nm}$ in diameter. Two different batches were produced: red SPs made of CQDs emitting at $630 \pm 5 \mathrm{~nm}$ and SPs with a blend of red and green CQDs at a ratio of 3:1, with the green CQDs emitting at $540 \pm 5 \mathrm{~nm}$. Resulting SPs dispersed in water were drop-cast onto a glass substrate and, once the water evaporated, optically-pumped individually.

\section{B. Optical Characterization}

SPs were selected and optically pumped one at a time with a $0.76 \mathrm{~ns}$ pulse width microchip pulsed laser $(\lambda=532 \mathrm{~nm})$ at a repetition rate of $7.1 \mathrm{kHz}$ and with a beam spot area of approximately $2.88 \times 10^{-7} \mathrm{~cm}^{2}$. The beam intensity was controlled by a variable wheel neutral density attenuator and focused on the sample with an objective lens (N PLAN EPI 50 $\times /$ 0.75, Leica). A spectrometer (AvaSpec-2048-4-DT, Avantes) was used to acquire the spectral data [4]. The size of the SPs was measured optically with a microscope and verified by the pseudo-Free Spectral Range (pseudo FSR) of their cavities, $\Delta v_{n, l}^{\Delta l} \approx \frac{c}{2 \pi . \mathrm{N} . a}$, where the radius $a$ of a sphere with refractive index $N$ can be expressed by the spacing between two consecutive modes with the same polarization [1]. The Q-factor, $Q=\frac{\lambda}{\Delta \lambda}$, was calculated from the resonance center wavelength, $\lambda$, and the resonance peak width at full width half maximum, $\Delta \lambda$, below lasing threshold [1].

\section{RESULTS AND DISCUSSION}

Two SPs (both $6.0 \pm 0.5 \mu \mathrm{m}$ in diameter) were selected from the synthesized batches, one containing exclusively red CQDs (red SP) and the other containing the blend of CQDs (blended SP). These two SPs were then characterized, and their Q-factors and laser intensity transfer functions compared. Emission below and above lasing threshold of the red SP is shown in Fig. 1. The red SP had its Q-factor estimated from the modes highlighted in grey on the normalized emission below threshold and its Fourier transform

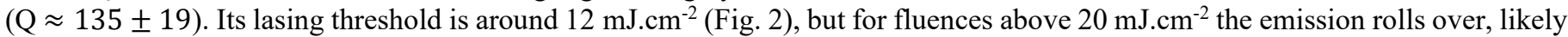
due to electron-hole separation, charge diffusion and nonradiative recombination [2]. Micrographs of the red SP below and above threshold are shown in Fig. 3. Emission data above and below lasing threshold for the blended SP are shown in Figs. 4, 5 and 6. The presence of green CQDs reduces the density of red CQDs and therefore diminishes exciton diffusion and self-absorption at the laser transition, leading to an enhanced $\mathrm{Q}$-factor $(\mathrm{Q} \approx 340 \pm 60)$. The higher $\mathrm{Q}$ is also reflected in the lower slope above threshold (Fig. 5). The effect of a lower density of the red CQDs on the modal gain is not only mitigated by the lower exciton diffusion but 
also by the fact that green CQD can act as donors. In turn the lasing threshold is not affected significantly $\left(12-14 \mathrm{~mJ}^{\mathrm{cm}} \mathrm{cm}^{-2}\right)$, and the SP laser is able operate at higher intensity without rolling over (Fig. 5).

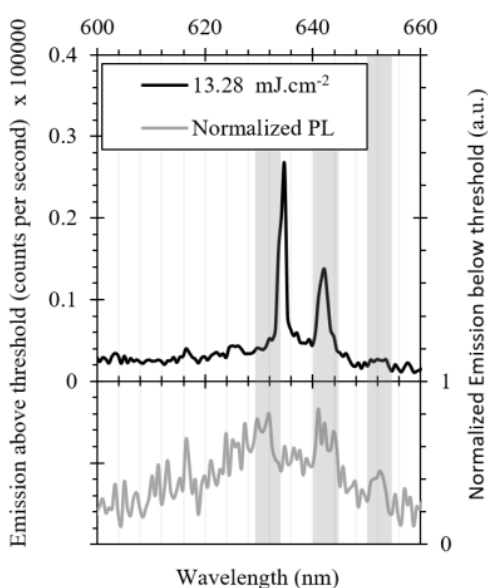

Fig. 1. Emission of the red SP above $\left(13.3 \mathrm{~mJ} . \mathrm{cm}^{-2}\right)$ and below (normalized PL) lasing threshold.

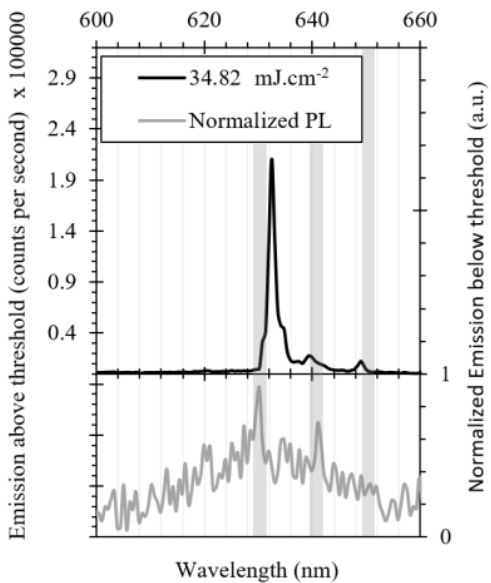

Fig. 4. Emission of the blended SP above $\left(34.8{\left.\mathrm{~mJ} . \mathrm{cm}^{-2}\right)}^{-2}\right.$ and below (normalized PL) lasing threshold.

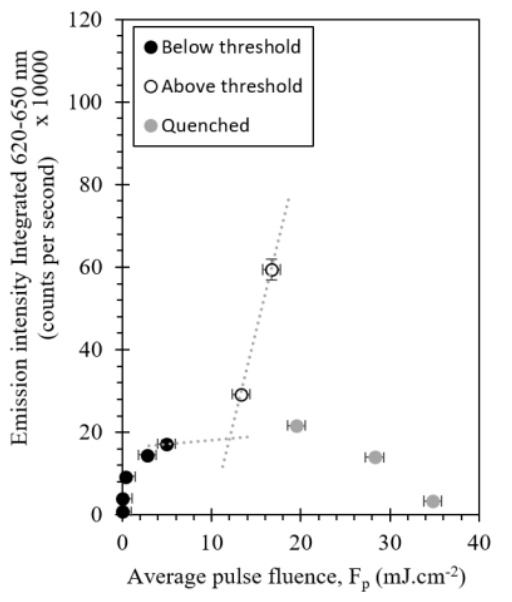

Fig. 2. Laser transfer function of the red SP.

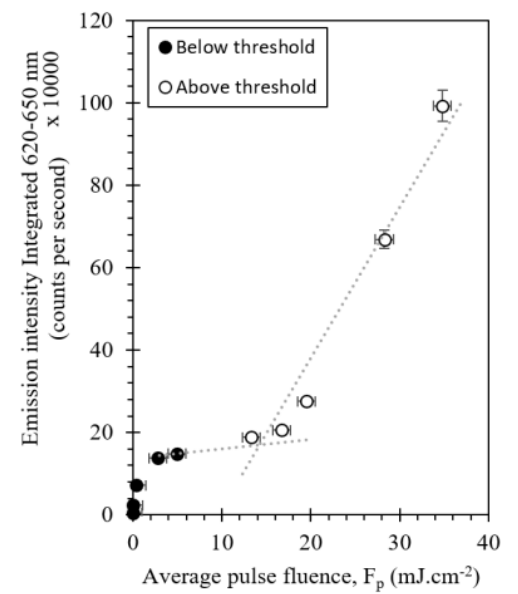

Fig. 5. Laser transfer function of the blended SP.
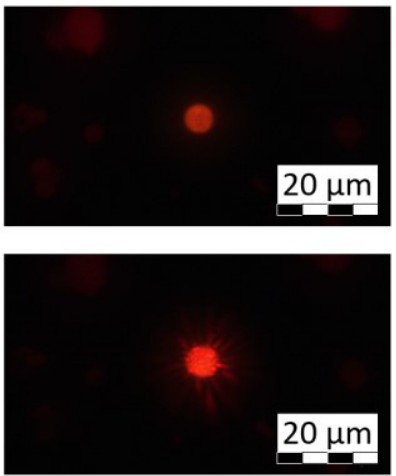

Fig. 3. Microscope images of the red SP below and above lasing threshold.
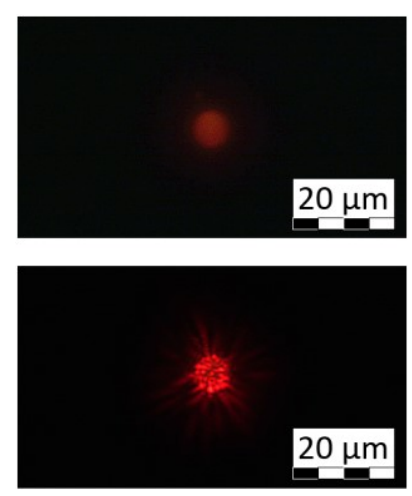

Fig. 6. Microscope images of the blended SP below and above lasing threshold.

\section{CONCLUSIONS}

Blending CQDs of two different bandgaps has been shown to improve the Q-factor and emission intensity of self-assembled microsphere CQD lasers. This work is an important step towards the design of higher quality CQD microcavities.

\section{ACKNOWLEDGMENT}

We acknowledge support from the EPSRC Platform Grant EP/P0274X/2 and the Leverhulme Trust for the Research Leadership Award RL-2019-038. The dataset can be found at: https://doi.org/10.15129/0517de89-0eda-4932-90b9-6655c8035a76.

\section{REFERENCES}

[1] G. C. Righini et al., "Whispering Gallery Mode microresonators: Fundamentals and applications," Riv. del Nuovo Cim., vol. 34, no. 7, pp. 435-488, 2011.

[2] N. Kholmicheva, P. Moroz, H. Eckard, G. Jensen, and M. Zamkov, "Energy transfer in quantum dot solids," ACS Energy Lett., vol. 2, no. 1, pp. 154-160, 2017.

[3] E. Marino et al., "Favoring the Growth of High-Quality, Three-Dimensional Supercrystals of Nanocrystals," J. Phys. Chem. C, vol. 124, no. 20, pp. 11256-11264, 2020.

[4] P. U. Alves, N. Laurand, and M. D. Dawson, "Multicolor laser oscillation in a single self-assembled colloidal quantum dot microsphere," 2020 IEEE Photonics Conf. IPC 2020 - Proc., pp. 2020-2021, 2020. 